[15] Prakash, P., Nayak, M. K., Chauhan, A. K. (2017). P-selectin can promote thrombus propagation independently of both von Willebrand factor and thrombospondin-1 in mice. Journal of Thrombosis and Haemostasis, 15 (2), 388-394. doi: http:// doi.org/10.1111/jth.13586

[16] Ivanov, I. I., Apta, B. H. R., Bonna, A. M., Harper, M. T. (2019). Platelet P-selectin triggers rapid surface exposure of tissue factor in monocytes. Scientific Reports, 9 (1). doi: http://doi.org/10.1038/s41598-019-49635-7

[17] Suthahar, N., Meijers, W. C., Silljé, H. H. W., Ho, J. E., Liu, F.-T., de Boer, R. A. (2018). Galectin-3 Activation and Inhibition in Heart Failure and Cardiovascular Disease: An Update. Theranostics, 8 (3), 593-609. doi: http://doi.org/10.7150/thno.22196

[18] Hogas, S., Bilha, S. C., Branisteanu, D., Hogas, M., Gaipov, A., Kanbay, M., Covic, A. (2017). Potential novel biomarkers of cardiovascular dysfunction and disease: cardiotrophin-1, adipokines and galectin-3. Archives of Medical Science, 4, 897-913. doi: http://doi.org/10.5114/aoms.2016.58664

[19] Berezin, A. E., Berezin, A. A. (2020). Circulating Cardiac Biomarkers in Diabetes Mellitus: A New Dawn for Risk Stratification - A Narrative Review. Diabetes Therapy, 11 (6), 1271-1291. doi: http://doi.org/10.1007/s13300-020-00835-9

[20] Lorenzo-Almorós, A., Pello, A., Aceña, Á., Martínez-Milla, J., González-Lorenzo, Ó., Tarín, N. et. al. (2020). Galectin-3 is Associated with Cardiovascular Events in Post-Acute Coronary Syndrome Patients with Type-2 Diabetes. Journal of Clinical Medicine, 9 (4), 1105. doi: http://doi.org/10.3390/jcm9041105

\title{
CURRENT TRENDS IN THE PREVALENCE OF FOOD HYPERSENSITIVITY IN CHILDREN OF THE KYIV REGION OF UKRAINE
}

\author{
Liudmyla Duda \\ Department of Pediatrics No. 1 \\ Shupyk National Medical Academy of Postgraduate Education \\ 9 Dorogozhytska str., Kyiv, Ukraine, 04112 \\ luda.duda@gmail.com
}

Abstract

The aim of the study. Due to the growing prevalence of food hypersensitivity worldwide and the number of dangerous food reactions, the aim was to determine the current prevalence of food hypersensitivity symptoms among children in the Kyiv region, Ukraine.

Methods. Total of 7106 children were surveyed: Group I (4 months - 5 years) had 1787 children, Group II (6-7 years) 2080 children, Group III (13-14 years) - 1909 children, Group IV (15-17 years) - 1330 children.

Results. The prevalence of food hypersensitivity among the surveyed children is $26.5 \%$ (95\% CI: $25.5-27.6)-$ this is 1888 children out of 7106. Highest prevalence of food hypersensitivity (FHS) is observed in the Group I, which is $32 \%$ (95\% CI: 29.8-34.2) - 571/1787, Groups II - 27.2 \% (95 \% CI: 25.7-29.8) - 566/2080, Groups III - $27.7 \%$ (95 \% CI: 14.7-18.0) - 529/1909, in the Group IV - 16.7 \% (95 \% CI: 25.3-29.2) - 222/1330). FHS in children is most often manifested by skin symptoms, followed by gastrointestinal manifestations, and acute urticaria/angioneurotic edema is at the third place. Respiratory manifestations possess $5 \%$ in the structure of FHS symptoms. An anaphylactic reaction was observed in 4 children out of 7106 respondents.

Conclusions. The prevalence of food hypersensitivity among Ukrainian children is high. There is an increase in the prevalence of urticaria/angioedema in preschool children.

Keywords: epidemiology, the prevalence of food hypersensitivity, food allergy, children, urticaria. 


\section{Introduction}

The food allergies prevalence rate worldwide is from $4-8 \%$ to $10 \%[1,2]$. According to a study conducted in the United States based on the study of individual primary care cards the prevalence of food allergies was $6.7 \%$, with the peak prevalence ranging from 0 to 3 years of age (5.7\%) [3]. The prevalence of food hypersensitivity in the UK (for both IgE-mediated food allergy and non IgE-mediated food hypersensitivity in early childhood) was $5.0 \%(3.7-6.7)$ [4]. The overall rate of food allergy was estimated $7.1 \%$ for children in Canada in a population-based self-report study using random digit telephone sampling and adjusting for non-response [5]. In China, 534 of 4151 parents completed screening questionnaires, have indicated that their children had diseases or problems caused by certain foods or certain types of food; therefore, the parent-reported rate of food allergy was $12.86 \%$ [6]. Estimated rates of food allergy prevalence vary widely, probably because of differences in study methodology including use of different definitions of food allergy, and different studied geographic area [5,7]. It has been increasing in the past twenty years and it represents a major public health problem in industrialized countries [8, 9]. The highest rates are registered in economically and socially developed countries, such as the United States, Western Europe, Australia, and Asia [10, 11]. Experts are concerned about environmental change, globalization and global industrialization, including the impact on the food industry, as not only the prevalence of food allergies is increasing, but the risk of severe reactions is also increasing [1].

Adverse reactions to foods combine the immune reactions, which include food allergy, as well as food intolerances (food intolerance of certain products or their components, which develops by non-immunological mechanisms) $[12,13]$. Food allergy is very distinct from food intolerance, which is defined as a non-immune reaction that includes metabolic, toxic, pharmacologic, and undefined mechanisms [14]. A food allergy is an immunoglobulin E (IgE)-mediated hypersensitive reaction to food, which consists in the appearance of allergic symptoms; it can vary from common urticaria to even fatal anaphylaxis $[3,15]$. Food allergy is not one disease but a spectrum of clinicopathological disorders [14]. Diseases associated with immune-mediated food allergy include acute urticaria/angioedema, oral allergy syndrome, atopic dermatitis, eosinophilic gastrointestinal disease, allergic contact dermatitis, and food protein-induced enteropathy/enterocolitis syndrome. Although celiac disease is an immune-mediated disease triggered by gluten, a food protein, it is not typically classified as a food-allergic disease. Manifestations of food allergies differ significantly, depending on the immune mechanism involved and the affected target organ [14]. Food allergies often are overreported because they may be confused with food intolerances or nonimmunologic adverse food reactions (e. g., lactose intolerance, food poisoning, caffeine intolerance) [16].

The World Allergy Organization (WAO) has proposed a new nomenclature since 2003, according to which allergy is a hypersensitivity reaction initiated by specific immunological mechanisms. If other mechanisms can be proven, the term non-allergic hypersensitivity should be used [17]. Sensitization is defined as the state of having detectable food-specific IgE which can be a precursor to the development of clinical food allergy [14]. The term hypersensitivity should be used to describe objectively reproducible symptoms or signs caused by certain stimuli that are well tolerated by healthy people. On the contrary, intolerance describes a distorted physiological response to an agent, which may be certain foods or supplements. Thus, the term food hypersensitivity is more general.

The difficulty of identifying adverse reactions to food and the variability of clinical manifestations due to both immune mediated (food allergy and celiac disease) and non-immune mediated (primarily food intolerances) reactions significantly affect statistics, particularly in Ukraine. Patients, doctors and other health professionals frequently confuse non-allergic food reactions with food allergy.

To date, there is very little data on the prevalence of food hypersensitivity among Ukrainian children, there are only a few studies. Thus, according to a study conducted in Ukraine in Zaporizhzhia and Zaporizhzhia region, colleagues note that the prevalence of food hypersensitivity in the region varies from $0.1 \%$ to $16.2 \%$. The structure of the respondents was dominated by children under three years [18]. There is a need for thorough epidemiological studies in Ukraine, and the implementation of standardized epidemiological studies of the prevalence of food hypersensitivity 
in accordance with international standards is still relevant. The true prevalence of food allergy remains elusive.

That is why the purpose of the fragment of our study was to determine the current level of prevalence of food hypersensitivity (FHS) symptoms among children in the Kiev region.

\section{Materials and methods}

Since the term FHS is more general, we will use it in our study, as at the stage of the questionnaire we will refer to the data provided by respondents without proper diagnostic evaluation.

To study the prevalence of allergy diseases (AD), we used the standardized questionnaires of the international program ISAAC (International Study of Asthma and Allergies in Childhood) with the expansion of age categories and nosologies, based on EAACI (European Academy of Allergy, Asthma and Clinical Immunology) guidelines. To study the prevalence of FHS, the questionnaire was supplemented with questions that contained the characteristic symptoms of this disease.

During 2017-2019, survey was conducted at pre-schools and schools of the Kyiv region among children of the following age categories: 4 months - 5 years (Group I), 6-7 years (Group II), 13-14 years (Group III), and 15-17 years (Group IV). Group II and Group III are chosen according to the recommendations of the ISAAC program. Group I and Group IV were selected by us additionally to study the prevalence of AD in children of these age categories. Children under 5 years combined infancy, early childhood and preschool categories, as the number of respondents was too little for separation in each category. At this age, the setting and formation of AD occur, and the diagnosis of most $\mathrm{AD}$ in children of the first years of life remains unrecognized. Children of 15-17 years old are a vulnerable and little controlled by parents and, accordingly, doctors category due to psychological and neuro-physiological features of age. In accordance to the ISAAC program methodology, to obtain valid results, the sample size of 3000 children for each age group was recommended (in case of individual centers study, a minimum sample of 1000 people was allowed). To establish a representative sample size, we used the formula for determining the sample size by Lisitsyn Yu. P. (1987), according to which the optimal sample size was 7105 children [19].

The study was conducted in compliance with the current Ukrainian legislation and meets the international ethical requirements. The approval of bioethical expert commission was received. The permits issued by the Department of Education and Science and the Department of Health of the Kyiv region were obtained. Written questionnaires were completed by children's parents and by senior students on a voluntary basis. Educational institutions have been chosen randomly [19].

Data analysis was performed using "R" version 3.6.0. Statistical analysis was performed for discrete data. $95 \%$ confidence intervals (CI) were calculated to determine the prevalence of symptomatic manifestations of AZ. For qualitative variables, the chi-squared test was used.

\section{Results}

As a result of the study, 7106 correctly completed questionnaires were received at this stage, which is $98.6 \%$ of the respondents. The distribution by the age categories is following: Group I had 1787 children, Group II - 2080 children, Group III - 1909 children, Group IV - 1330 children. The gender distribution of the sample is about 1:1 [19].

According to the results of the study, the prevalence of food hypersensitivity among the surveyed children is $26.5 \%$ (95\% CI: $25.5-27.6)$ - this is 1888 children out of 7106 , one in four children had symptoms of FHS. Of these, among girls - 25.5 \% (95\% CI: 24.1-27.7) - 940/3688, and among boys $-27.7 \%$ (95\% CI: 26.2-29.3) - 948/3418, with a significant difference by sex $\left(\chi^{2}=4,478 ; p=0,034\right)$. The highest prevalence of FHS is observed in the Group I (4 months-5 years), which is $32 \%$ (95 \% CI: 29.8-34.2) - 571/1787, and in the Group IV (15-17 years) the level of food hypersensitivity is already halved, but the indicators remain quite high $-16.7 \%$ (95 \% CI: $25.3-29.2)-222 / 1330$, with a significant difference between groups $\left(\chi^{2}=94.774 ; p=0.000\right)$. In the Groups II and III the indicators are $27.2 \%$ (95 \% CI: $25.7-29.8)-566 / 2080$ and $27.7 \%$ (95 \% CI: 14.7-18.0) - 529/1909, respectively, without a significant difference between them. There is an age-related tendency to a gradual decrease in the prevalence of FHS, which is primarily due to anatomical and physiological age characteristics (Fig. 1). 


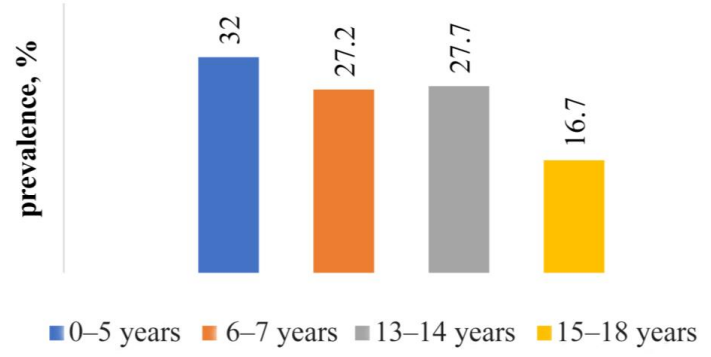

Fig. 1. FHS Prevalence by age groups among the children of Kyiv region

Considering the prevalence of FHS by age structure of respondents, without grouping them, and then analyzing the trend line, the highest rates are noted among children under one year, which is $40.7 \%(11 / 27)$ (without a significant difference, probably due to a small number of children under one year who participated in the study); among children from one to two years - $25.7 \%(96 / 373)$; against the background of the general tendency to a gradual decrease in indicators with age, their range is kept at the level of $25-35 \%$, i.e. one third of children from 2 to 14 years have symptoms of FHS; in adolescence there is a decline below $20 \%$ from $18.4 \%$ (from the age of 15 ) to $12.9 \%$ (in 17-year-old children).

FHS manifests in a variety of clinical conditions within the gastrointestinal tract, skin and lungs, with the most dramatic and sometimes fatal manifestation being anaphylactic shock. When analyzing the obtained survey data, there was a need to group the symptoms. The following groups were identified: skin (skin rash of various kinds with itching due to food consumption, but this group of symptoms did not include urticaria/angioedema), gastrointestinal manifestations (nausea, vomiting, abdominal pain, stool disorders, abnormal stools), respiratory (rhinitis, cough, shortness of breath, bronchospasm), manifestations in the form of urticaria/angioedema as well as a separate reaction to food in the form of anaphylactic reaction were distinguished into separate groups.

The prevalence of FHS symptoms, extrapolated to the population, is: skin $-20.5 \%$ (95 \% CI: 19.6-21.5) - 1459/7106; gastrointestinal - $9.3 \%$ (95 \% CI: 8.6-9.9) - 659/7106; respiratory - $1.7 \%$ (95 \% CI: 1.5-2.1) - 125/7106; urticaria/angioedema - 3.7 \% (95 \% CI: 3.3-4.2) - 264/7106 (Fig. 2). An anaphylactic reaction was observed in 4 children out of 7106 respondents (caused by fish, peanuts, peach) $-0.001 \%$ (95\% CI: $0-0.001)$.

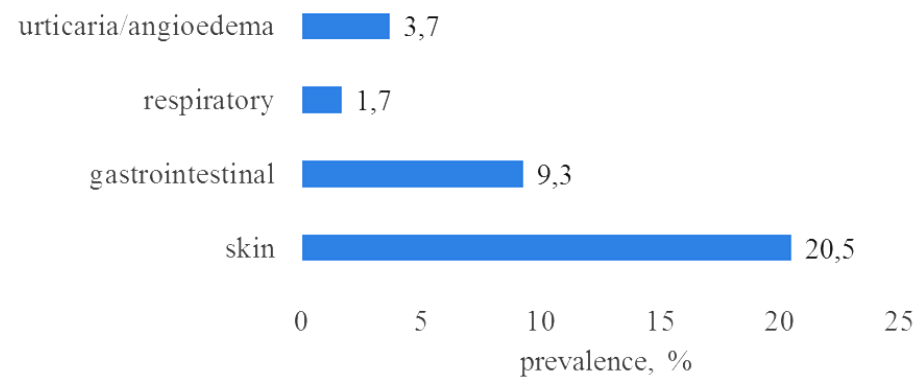

Fig. 2. The structure of FHS symptoms in children

The highest prevalence rates of skin and gastrointestinal symptoms were observed in Group I with a gradual decrease by half in Group IV (Table 1). Respiratory manifestations with the highest rates are observed among adolescents of $13-14$ years $\left(\chi^{2}=13.86 ; p=0.003\right)$, which is known as the most vulnerable age category both from the standpoint of physiological (hormonal, autonomic) and psychological features, which requires our targeted attention.

The lowest prevalence of skin manifestations in Group IV (15-17 years) can be explained by the evolution of atopic dermatitis with age into the syndrome of constitutionally dry skin, which is often no longer considered the disease.

Regarding the gastrointestinal manifestations of FHS, it should be noted that in adolescents (Group III and IV) these symptoms are masked by functional disorders of the gastrointes- 
tinal tract or become chronic forms of various gastrointestinal pathologies, so such children are redistributed between different specialists and, accordingly, they themselves do not perceive it as a manifestation of allergies [20]. We assume that these figures may be much higher, which requires further research.

Table 1

Prevalence of food hypersensitivity symptoms among children of Kyiv region, distribution by age groups, n/\%

\begin{tabular}{|c|c|c|c|c|c|}
\hline \multirow{3}{*}{ FHS Symptoms } & \multicolumn{4}{|c|}{ Age groups } & \multirow{3}{*}{$\mathbf{p}^{*}$} \\
\hline & I & II & III & IV & \\
\hline & $(n=1787)$ & $(n=2080)$ & $(n=1909)$ & $(n=1330)$ & \\
\hline Skin & $475 / 26.6$ & $440 / 21.2$ & $373 / 19.5$ & $171 / 12.9$ & $<0.001$ \\
\hline Gastrointestinal & $200 / 11.2$ & $198 / 9.5$ & $178 / 9.3$ & $83 / 6.2$ & $<0.001$ \\
\hline Urticaria/angioedema & $70 / 3.9$ & $72 / 3.5$ & $34 / 1.8$ & $88 / 6.6$ & $<0.001$ \\
\hline Respiratory & $24 / 1.3$ & $39 / 1.9$ & $49 / 2.6$ & $13 / 1.0$ & $<0.001$ \\
\hline
\end{tabular}

Note: *-statistically significant difference between groups

It should be noted that there is a rejuvenation of the manifestations of FHS in the form of urticaria/angioedema. Urticaria/angioedema as adverse reactions to foods can be considered IgE-mediated food allergy. There are quite high rates of urticaria/angioedema caused by food in Group I children, which is $3.9 \%$ (70/1787) [13]. Significantly the highest prevalence of urticaria/ angioedema among age groups $\left(\chi^{2}=51.84 ; \mathrm{p}=0.000\right)$ are observed in children of $15-18$ years (Group IV) - $6.6 \%$ (88/1330).

Significantly higher rates among boys are observed in group I, namely manifestations of FHS in the form of urticaria/angioedema, in Group II - respiratory manifestations, in Group III - gastrointestinal manifestations. The distribution of FHS symptoms prevalence by sex is given in Table 2 .

Table 2

The prevalence of FHS symptoms, distribution by Groups and sex (\%; $95 \% \mathrm{CI})$

\begin{tabular}{cccccc}
\hline \multirow{2}{*}{ Age Groups } & & \multicolumn{4}{c}{ Symptoms of food hypersensitivity } \\
\cline { 3 - 5 } & & Skin & Gastrointestinal & Respiratory & Urticaria/angioedema \\
\hline \multirow{2}{*}{ I (n=1787) } & F & $25.2 ; 22.4-28.2$ & $10.6 ; 8.6-12.8$ & $1.0 ; 0.5-1.9$ & $3.0 ; 1.9-4.3$ \\
& M & $27.9 ; 25-30.9$ & $11.8 ; 9.8-14.1$ & $1.7 ; 0.9-2.7$ & $4.9 ; 3.5-6.5^{*}$ \\
II (n=2080) & F & $20.4 ; 18-23$ & $8.8 ; 7.1-10.7$ & $1.2 ; 0.6-2.1$ & $2.9 ; 1.9-4.1$ \\
& M & $21.8 ; 19.4-24.4$ & $10.2 ; 8.4-12.1$ & $2.5 ; 1.7-3.6^{*}$ & $4.0 ; 2.9-5.4$ \\
III (n=1909) & F & $18.9 ; 16.6-21.5$ & $7.8 ; 6.2-9.6$ & $1.9 ; 1.1-2.9$ & $1.2 ; 0.6-2.1$ \\
& M & $20.2 ; 17.6-23.0$ & $11.0 ; 9.1-13.3 *$ & $3.3 ; 2.2-4.7$ & $2.4 ; 1.5-3.7$ \\
IV (n=1330) & F & $14.2 ; 11.8-16.8$ & $6.4 ; 4.8-8.3$ & $0.6 ; 0.2-1.5$ & $7.2 ; 5.5-9.2$
\end{tabular}

Note: ${ }^{*}-p \leq 0.05$ (statistically significant difference)

\section{Discussion}

Food allergy is a significant clinical problem in Ukraine. Received results demonstrate high prevalence of food allergy, which is consistent with worldwide trends [2, 4]. It is hard to compare data from Ukraine due to quite low range of FHS studies in Ukraine. 
Considering the study design (questionnaires) and keeping in mind that the term "food hypersensitivity" is more wide we have relied on parent' and children's subjective data, requiring further clarification with possible additional examinations and clarification of diagnosis, which is planned for further stage of our study. We realize that provided evidences of FHS prevalence include immune IgE-mediated and non-IgE-mediated as well as non-immunological food reactions. But received results are quite important and point to significant problem of food allergy and its scope in Kyiv region as the most populous region of Ukraine, and also reveals the difficulties in diagnosing, particularly due to terminology. Since the diagnosis of food allergy reactions in children remains enough difficult, respectively, there is certain variability in the results of different studies of food allergy and food hypersensitivity prevalence. It should be noted that symptoms of FHS, such as skin, gastrointestinal, and respiratory, need further study to clarify the options for immune responses, including food allergies and food intolerance, which are often associated with various functional disorders in children.

Limitations of the study. The results of our study are significant addition to already completed studies, but the studying of food allergy prevalence in Ukraine remains insufficient. Additionally, there are regional differences of food allergens could be due to genetic or environmental factors. Additional research is needed in different regions of Ukraine to determine whether epidemiological trends are changing.

Further research is needed to improve the diagnostic criteria and verification of the diagnosis of food allergy for more effective prevention and treatment of food allergy.

\section{Conclusion}

1. The prevalence of FHS symptoms in children of Kyiv region of Ukraine is quite high $26.5 \%$ (95 \% CI: 25.5-27.6).

2. Skin symptoms in the structure of FHS take the first place, the prevalence of which is $20.5 \%$ (95 \% CI: 19.6-21.5), and the second place is occupied by gastrointestinal symptoms $9.3 \%$ (95 \% CI: 8.6-9.9).

3. The highest prevalence of respiratory symptoms of FHS is observed at the age of 13-14 years.

4. IgE-mediated food allergy in the form of urticaria/angioneurotic edema is increasingly recognized as the most common in preschool children.

5. The highest prevalence of urticaria/angioneurotic edema among age groups is observed in children aged $15-18$ years $(6.6 \%)$.

\section{Conflict of interests}

The authors declare that they have no conflicts of interest.

\section{References}

[1] Burks, A. W., Tang, M., Sicherer, S., Muraro, A., Eigenmann, P. A., Ebisawa, M. et. al. (2012). ICON: Food allergy. Journal of Allergy and Clinical Immunology, 129 (4), 906-920. doi: http://doi.org/10.1016/j.jaci.2012.02.001

[2] Devdas, J. M., Mckie, C., Fox, A. T., Ratageri, V. H. (2017). Food Allergy in Children: An Overview. The Indian Journal of Pediatrics, 85 (5), 369-374. doi: http://doi.org/10.1007/s12098-017-2535-6

[3] Hill, D. A., Grundmeier, R. W., Ram, G., Spergel, J. M. (2016). The epidemiologic characteristics of healthcare provider-diagnosed eczema, asthma, allergic rhinitis, and food allergy in children: a retrospective cohort study. BMC Pediatrics, 16 (1). doi: http://doi.org/10.1186/s12887-016-0673-z

[4] Grimshaw, K. E. C., Bryant, T., Oliver, E. M., Martin, J., Maskell, J., Kemp, T. et. al. (2015). Incidence and risk factors for food hypersensitivity in UK infants: results from a birth cohort study. Clinical and Translational Allergy, 6 (1). doi: http:// doi.org/10.1186/s13601-016-0089-8

[5] Savage, J., Johns, C. B. (2015). Food allergy: epidemiology and natural history. Immunology and allergy clinics of North America, 35 (1), 45-59. doi: http://doi.org/10.1016/j.iac.2014.09.004

[6] Dai, H., Wang, F., Wang, L., Wan, J., Xiang, Q., Zhang, H. et. al. (2020). An epidemiological investigation of food allergy among children aged 3 to 6 in an urban area of Wenzhou, China. BMC Pediatrics, 20 (1). doi: http://oi.org/10.1186/s12887020-02115-8 
[7] Iweala, O. I., Choudhary, S. K., Commins, S. P. (2018). Food Allergy. Current Gastroenterology Reports, 20 (5). doi: http:// doi.org/10.1007/s11894-018-0624-y

[8] Ebisawa, M., Ito, K., Fujisawa, T. (2017). Japanese guidelines for food allergy 2017. Allergology International, 66 (2), $248-264$. doi: http://doi.org/10.1016/j.alit.2017.02.001

[9] Barni, S., Liccioli, G., Sarti, L., Giovannini, M., Novembre, E., Mori, F. (2020). Immunoglobulin E (IgE)-Mediated Food Allergy in Children: Epidemiology, Pathogenesis, Diagnosis, Prevention, and Management. Medicina, 56 (3), 111. doi: http:// doi.org/10.3390/medicina56030111

[10] Food allergy in children and young people (2011). NICE clinical guideline 116. Available at: http://www.nice.org.uk/guidance/ cg116/evidence/full-guideline-136470061

[11] Le, T. T. K., Nguyen, D. H., Vu, A. T. L., Ruethers, T., Taki, A. C., Lopata, A. L. (2019). A cross-sectional, population-based study on the prevalence of food allergies among children in two different socio-economic regions of Vietnam. Pediatric Allergy and Immunology, 30 (3), 348-355. doi: http://doi.org/10.1111/pai.13022

[12] Turnbull, J. L., Adams, H. N., Gorard, D. A. (2014). Review article: the diagnosis and management of food allergy and food intolerances. Alimentary Pharmacology \& Therapeutics, 41 (1), 3-25. doi: http://doi.org/10.1111/apt.12984

[13] Shadrin, O. G., Nyankosvkyi, S. L., Dobrianskyi, D. O., Bielousova, O. Yu., Nedelska, S. M., Ivakhnenko, O. S. et. al. (2014). Osoblyvosti diagnostyky ta pidkhody do likuvalno-profilaktychnogo kharchuvannya ditei ranniogo viku z alergiyeyu do bilka korovyachogo moloka. Kyiv, 28.

[14] Anvari, S., Miller, J., Yeh, C.-Y., Davis, C. M. (2018). IgE-Mediated Food Allergy. Clinical Reviews in Allergy \& Immunology, 57 (2), 244-260. doi: http://doi.org/10.1007/s12016-018-8710-3

[15] Johnston, L. K., Chien, K. B., Bryce, P. J. (2014). The Immunology of Food Allergy. The Journal of Immunology, 192 (6), 2529-2534. doi: http://doi.org/10.4049/jimmunol.1303026

[16] Mehta, R. (2018) Allergy and Asthma: Food Allergies. FP Essent, 472, 16-19.

[17] Baral, V., O’B Hourihane, J. (2005). Food allergy in children. Postgraduate Medical Journal, 81 (961), 693-701. doi: http:// doi.org/10.1136/pgmj.2004.030288

[18] Pakholchuk, O. P., Nedelskaya, S. M. (2017). Porivnyalna kharakterystyka metodiv diagnostyky giperchutlyvosti do kharchovykh produktiv u ditey. Astma ta alergiya, 2, 2017.

[19] Duda L. V. (2019). Trends in prevalence of asthma in children by the example of the kyiv region of Ukraine. World Science, 2 (11 (51)), 12-16. doi: http://doi.org/10.31435/rsglobal_ws/30112019/6769

[20] Duda, L. V., Okhotnikova, E. N. (2018). Clinical and epidemiological characteristics of the most common allergy diseases in children. CHILD'S HEALTH, 13 (4), 345-355. doi: http://doi.org/10.22141/2224-0551.13.4.2018.137017 